\title{
PERFIL EPIDEMIOLÓGICO DA SÍFILIS GESTACIONAL: CLÍNICA E EVOLUÇÃO DE 2012 A 2017
}

EPIDEMIOLOGICAL PROFILE OF GESTATIONAL SYPHILIS: CLINIC AND EVOLUTION FROM 2012 TO 2017

PERFIL EPIDEMIOLÓGICO DE LA SÍFILIS GESTACIONAL: CLÍNICA Y EVOLUCIÓN DE 2012 A 2017

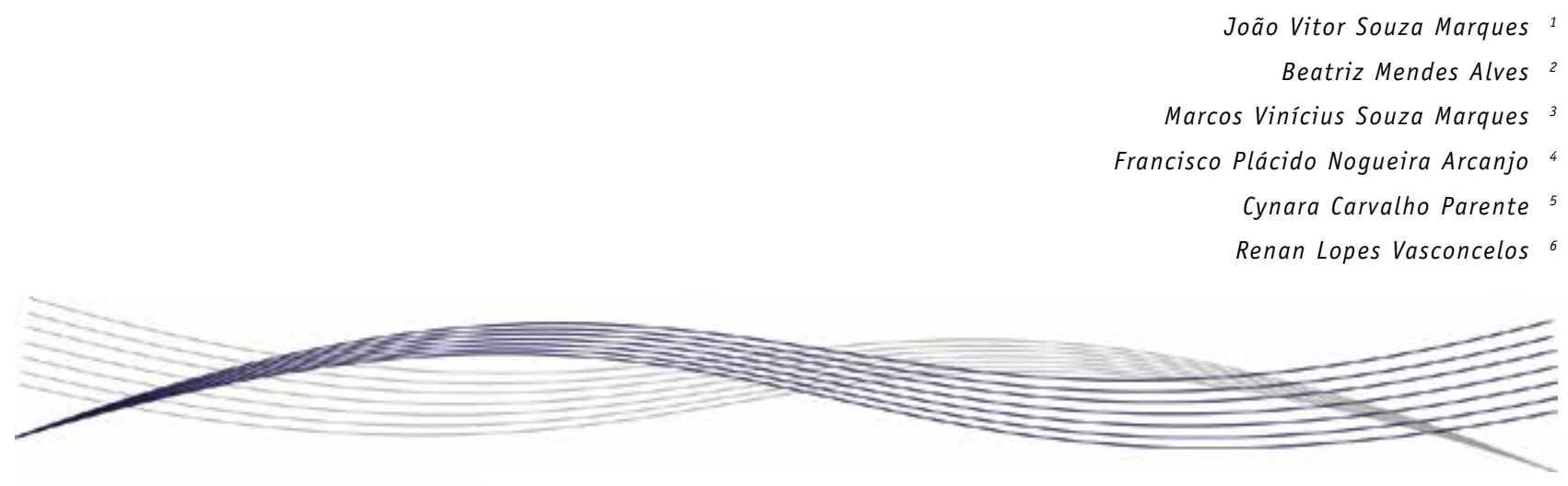

\section{RESUMO}

Palavras-chave:

Sifilis; Gestantes; Epidemiologia; Saúde Pública.

Keywords: Syphilis; Pregnant Women; Epidemiology; Public Health.

Palabras clave: Sífilis; Mujeres Embarazadas; Epidemiología; Salud Pública.

Submetido: $21 / 11 / 2018$

Aprovado: $03 / 12 / 2018$

Autor(a) para Correspondência: João Vitor Souza Marques Av. Dr. Guarani, 307, apto. 101 Derby Clube Sobral-CE CEP: 62042-030 E-mail: joao_vitorsm@hotmail.com
Este estudo teve por objetivo identificar aspectos clinicos e evolutivos da sifilis gestacional (SG) em Sobral-CE, a partir da análise do perfil epidemiológico no período de 2012 a 2017. Realizou-se coleta de dados no Sistema Nacional de Agravos de Notificação (Sinan). Cinco características epidemiológicas foram avaliadas: escolaridade; cor da pele; idade da paciente; zona de residência; e classificação clínica da doença. Um total de 452 casos da doença foi notificado no período de estudo, evidenciando aumento de notificações no município em comparação com a década passada. Constatou-se que 217 mulheres (48\%) tinham Ensino Fundamental incompleto, 403 (89,1\%) eram pardas ou negras, $336(74,4 \%)$ tinham entre 20 e 39 anos de idade, 377 (83,4\%) moravam na zona urbana do município e 341 dos casos $(75,4 \%)$ foram classificados como sifilis terciária. Concluiu-se que ações voltadas à prevenção, ao diagnóstico e ao tratamento da doença são importantes para reduzir o número de casos de SG em Sobral. Ademais, mostra-se necessário direcionar políticas sociais aos grupos vulneráveis, visando a diminuir os agravos dessa infecção.

\footnotetext{
1. Aluno de Graduação em Medicina na Universidade Federal do Ceará (UFC). Sobral (CE), Brasil.

2. Aluna de Graduação em Medicina da UFC. Sobral (CE), Brasil.

3. Aluno de Graduação em Medicina da UFC. Sobral (CE), Brasil.

4. Professor no Curso de Medicina da UFC. Sobral (CE), Brasil.

5. Professora no Curso de Medicina da UFC. Sobral (CE), Brasil.

6. Aluno de Graduação em Medicina da UFC. Sobral (CE), Brasil.
} 


\section{ABSTRACT}

This study aimed to identify clinical and evolutionary aspects of gestational syphilis (GS) in Sobral, Ceará, Brazil, by means of epidemiological profile analysis within the period from 2012 to 2017. Data collection was conducted in the Brazilian National System of Notifiable Diseases (SINAN). Five epidemiological characteristics were evaluated: schooling; skin color; patient's age; area of residence; and clinical classification of disease. A total of 452 cases of the disease were notified within the study period, evidencing an increased number of notifications in the municipality when compared to the previous decade. It was found that 217 women (48\%) had incomplete Elementary School, 403 (89.1\%) were brown or black-skinned, 336 (74.4\%) were between 20 and 39 years of age, $377(83.4 \%)$ lived in the urban area of the municipality, and 341 of the cases (75.4\%) were classified as tertiary syphilis. It was concluded that actions aimed at the prevention, diagnosis, and treatment of disease are significant to reduce the number of GS cases in Sobral. Also, there is a need to provide vulnerable groups with social policies, in order to decrease the complications of this infection.

\section{RESUMEN}

Este estudio tuvo como objetivo identificar los aspectos clínicos y evolutivos de la sifilis gestacional (SG) en Sobral, Ceará, Brasil, mediante el análisis del perfil epidemiológico en el período de 2012 a 2017. La recopilación de datos se realizó en el Sistema Nacional de Enfermedades Notificables de Brasil (SINAN). Se evaluaron cinco características epidemiológicas: escolarización; color de piel; edad del paciente; área de residencia; y clasificación clínica de la enfermedad. Un total de 452 casos de la enfermedad fueron notificados dentro del período de estudio, lo que evidencia un aumento en el número de notificaciones en el municipio en comparación con la década anterior. Se encontró que 217 mujeres (48\%) tenían Enseñanza Primaria incompleta, 403 (89,1\%) eran pardas o negras, 336 (74,4\%) tenían entre 20 y 39 años de edad, 377 (83,4\%) vivian en la zona urbana del municipio y 341 de los casos $(75,4 \%)$ se clasificaron como sifilis terciaria. Se concluyó que las acciones dirigidas a la prevención, al diagnóstico y al tratamiento de la enfermedad son importantes para reducir el número de casos de SG en Sobral. Además, existe la necesidad de proporcionar políticas sociales a los grupos vulnerables para disminuir las complicaciones de esta infección.

\section{INTRODUÇÃO}

A sífilis é uma infecção sexualmente transmissível (IST) causada pela bactéria Treponema pallidum, curável e exclusiva da raça humana. Apresenta-se em variadas manifestações clínicas e diferentes estágios (sífilis primária, secundária, latente e terciária), com maior risco de transmissão nas classificações primária e secundária. A sífilis pode ser transmitida durante uma relação sexual sem uso de preservativo ou para o(s) filho(s) durante a gestação ou o parto ${ }^{1}$.

A inclusão da sifilis gestacional (SG) como IST de notificação compulsória se justifica por sua alta prevalência e taxa de transmissão vertical (que pode variar de $30 \%$ a $100 \%$ se não tratada ou tratada inadequadamente). A SG é diagnosticada quando uma gestante apresenta sinais e sintomas clínicos de sífilis e/ou apresenta sorologia não treponêmica reagente, independente da titulação, mesmo na ausência de resultado de teste treponêmico, durante o pré-natal ou no momento da curetagem ou do parto².

A sífilis, se não tratada em uma fase precoce, pode acometer diversos órgãos do corpo, como o sistema nervoso, o coração, os ossos, a pele e os olhos. Seu período de incubação é bastante variável, com média de 3 semanas, variando de 10 a 90 dias. Essa infecção merece cuidado especial entre as gestantes em virtude da possibilidade de transmissão para o feto, causando repercussões clínicas importantes tanto no período gestacional como após o nascimento ${ }^{3}$.

Assim que a gestante for diagnosticada com sífilis, o tratamento imediato e adequado à fase clínica assume vital importância. A droga utilizada no tratamento da sífilis primária, secundária e latente precoce é a penicilina benzatina 2.400.000 UI IM, em dose única; na sífilis latente tardia ou indeterminada se administra 1 dose por semana da mesma droga, durante 3 semanas. Além disso, faz parte do tratamento realizar pesquisas para outras ISTs, proporcionar seguimento sorológico e, principalmente, tratar o parceiro, adotando abstinência sexual até curar a doença ${ }^{4}$.

Com vistas a estabelecer e avaliar a amplitude da SG e seus malefícios para o recém-nascido (RN) infectado, o Ministério da Saúde (MS) incluiu a 
SG e a sifilis congênita (SC) na lista nacional de doenças de notificação compulsória. Nesse sentido, - programa Rede Cegonha foi implantado para melhorar a atenção prestada à mãe e ao RN, de modo a aumentar o acesso a diagnóstico e à terapêutica da SG e SC no Brasil ${ }^{5-6}$.

0 diagnóstico e tratamento de SG são cruciais para a quebra da cadeia de transmissão da doença ao feto e/ou RN, pois, uma vez tratada adequadamente na gestante, as chances de transmissão vertical da doença diminuem consideravelmente, fato que comprova a necessidade de estudar a epidemiologia da SG. Assim, a subnotificação de casos de SG pode constituir fator de agravo e sequelas para o RN, com consequentes problemas de saúde pública. Um prénatal de qualidade deve notificar e tratar gestantes infectadas por sífilis para reduzir os índices de $\mathrm{SC}^{7}$.

Dados epidemiológicos recentes revelam um aumento de $300 \%$ do número de casos de SG no Brasil entre 2010 e 2016. Isso se deve, em parte, ao aumento do número de mulheres testadas por meio de testes rápidos e ao aumento do número de notificações da doença via Sistema Nacional de Agravos de Notificação (Sinan), contudo, acreditase que ainda exista um grande percentual de casos não notificados, cenário no qual a SG pode ser mais grave do que os dados indicam ${ }^{8}$.

$\mathrm{Na}$ última década houve um aumento de aproximadamente $370 \%$ dos casos de sífilis no Ceará. A sífilis adquirida, transmitida por relação sexual, mais comum na população entre 15 e 24 anos, aumentou $817 \%$, passando de 39 casos em 2010 para 358 em 2017. Já a SC aumentou cerca de $170 \%$ entre 2007 e 2015. A taxa de incidência no estado é de 8,7 casos para 1.000 nascidos vivos, taxa superior à do País, que é de 6,5 para 1.000 nascidos vivos, fato que comprova a necessidade de aumentar a atenção aos casos de sífilis, sobretudo a SG, nesse estado ${ }^{9}$.

Em Sobral-CE, a SG apresentou significativa variação nos últimos anos passou-se de 1 caso notificado em 2004 para 80 casos notificados em 2012. Também se deve atentar para possíveis subnotificações nesse período, fato que pode causar distorções no planejamento das políticas públicas de saúde por parte de gestores das três esferas de governo (municipal, estadual e federal). Diante disso, a perspectiva é de que a doença permanecerá em patamar semelhante ou mais grave nos próximos anos, o que demanda um novo olhar para esse problema de saúde coletiva ${ }^{10}$.

Com o agravamento do panorama da SG, avaliar o

\section{Na última década houve um aumento de aproximadamente $370 \%$ dos casos de sifilis no Ceará.}

perfil epidemiológico das pacientes infectadas tende a viabilizar a elaboração de estratégias de saúde que promovam a intervenção para o diagnóstico precoce dessa doença, em busca do aperfeiçoamento dos métodos de diagnóstico e tratamento disponíveis para lidar com essa clientela.

Diante do exposto, este artigo avalia o perfil epidemiológico de pacientes acometidas por SG em Sobral no período entre 2012 e 2017.

\section{METODOLOGIA}

Trata-se de pesquisa documental e retrospectiva, com abordagem quantitativa, realizada via coleta de dados na plataforma on-line Sinan. Houve avaliação de todos os casos de SG em Sobral no período entre 2012 e 2017.

Localizado no norte do Ceará, o município é considerado centro de convergência por sua ampla estrutura nos setores de saúde, comércio, educação, serviços e indústria. Sua população consistiu em 206.644 pessoas em 2018, com densidade demográfica de 88,67 habitantes por quilômetro ${ }^{2}$. Seu território tem 69 pontos de atendimento do Sistema único de Saúde (SUS), constituindo relevante polo de referência em serviços de saúde para dezenas de munícipios vizinhos, como Forquilha, Groaíras, Massapê, Santana do Acaraú, Cariré, Frecheirinha, Ipu, Varjota, Meruoca, Santa Quitéria, Mucambo e Alcântaras. Sobral apresenta um clima quente e seco, com temperatura média acima dos $30^{\circ} \mathrm{C}$, típico do bioma caatinga ${ }^{11-13}$.

As seguintes características epidemiológicas foram adotadas na análise do perfil das gestantes incluídas no estudo: escolaridade; cor da pele; idade da paciente; zona de residência; e classificação clínica da doença. Gestantes com sífilis que moram fora do país foram excluídas do estudo.

A coleta de dados foi realizada no programa computacional Microsoft Excel, versão 2010. Nesse 
sistema, as variáveis do estudo foram organizadas por ano, de modo a compreender o perfil temporal das infecções e a dinâmica do processo saúdedoença no município. Além disso, o programa computacional Microsoft Power Point, versão 2010, foi utilizado para a elaboração de gráficos que ilustram as características a discutir neste artigo. Por fim, a elaboração de tabelas agiliza e simplifica a visualização das variáveis sociodemográficas da SG.

Por utilizar o Sinan, plataforma on-line do MS cujos dados se encontram disponiveis para livre acesso, este estudo não demandou aprovação por comitê de ética em pesquisa com seres humanos.

\section{RESULTADOS E DISCUSSÃO}

De acordo com os dados do Sinan, Sobral apresentou um total de 452 casos de SG no período entre 2012 e 2017. A Figura 1 indica que foram notificados 80 casos em 2012 (17,7\%). 0 ápice foi atingido em 2013, com notificação de 86 casos $(19 \%)$, ao passo que 2014 teve 74 casos $(16,4 \%), 2015$ apresentou outros 80 casos $(17,7 \%)$ e 2016 e 2017 tiveram 66 casos cada $(14,6 \%)$. Isso demonstra aumento do número de casos em comparação a período de 2004 a 2011, quando foram notificados 231 casos no município ${ }^{10}$.

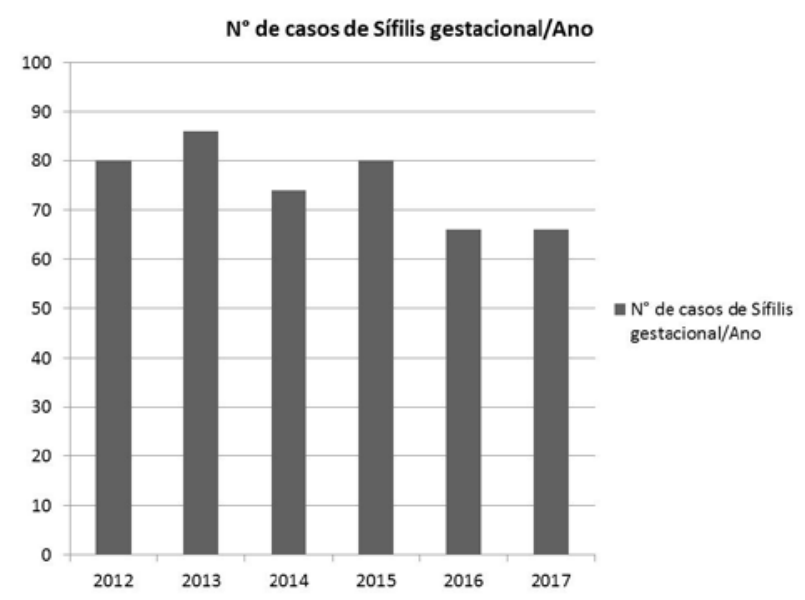

Figura 1. Número de casos de sífilis gestacional por ano. Sobral, 2012-2017.

Fonte: Elaborada pelos autores.

Umas das hipóteses desse aumento de casos se refere à ampliação do uso de testes rápidos em gestantes e a uma mudança comportamental, como diminuição do uso de métodos contraceptivos, sobretudo preservativos, como indicam os dados nacionais do Ministério da Saúde ${ }^{8}$.
0 alto número de casos notificados de SG demonstra que as medidas de prevenção têm sido ineficazes e acredita-se que a quantidade de gestantes com essa IST seja bem maior, devido à subnotificação de casos. Uma situação semelhante à descrita neste estudo foi observada em outro município da Região Nordeste: Santa Cruz, no Rio Grande do Norte ${ }^{14}$.

Soma-se ao exposto outro estudo, realizado no Rio de Janeiro, que analisa o panorama histórico da sífilis no Brasil e reitera o relato de aumento do número de casos dessa doença, em especial de SG. Tal realidade pode estar particularmente relacionada à falta de penicilina, medicamento adequado para a terapêtica, o que contribui para a manutenção da patologia ${ }^{15}$.

0s dados nacionais apontam a falta de medicamento em $60 \%$ dos estados no início do ano de 2016. Tal avaliação indica, ainda, cerca de 65.000 casos de sífilis adquirida no país em 2015, aproximadamente 33.000 deles de SG. Nesse contexto, a falta de medicamento é generalizada e parece refletir disputas de mercado para aumentar o preço do produto, aumentando sobremaneira a dificuldade da terapêutica dessa doença ${ }^{15}$.

A Tabela 1 mostra que, no período de estudo, houve 9 casos na faixa etária de 10 a 14 anos ( $2 \%$ do total), 94 casos de 15 a 19 anos $(20,8 \%), 336$ casos de 20 a 39 anos $(74,4 \%)$ e 13 casos de 40 a 59 anos $(2,8 \%)$. Tais dados indicam que a maioria dos casos de sífilis ocorre na faixa etária de 20 a 39 anos, reforçando a importância de programas de rastreio e notificação específicos para a fase de maior fertilidade das mulheres.

\section{0 alto número de casos notificados de SG demonstra que as medidas de prevenção têm sido ineficazes...}


Tabela 1 - Faixa etária dos casos de sífilis gestacional. Sobral, 2012-2017

\begin{tabular}{|c|c|c|c|c|c|}
\hline \multirow[b]{2}{*}{ Ano } & \multirow[b]{2}{*}{$10-14$} & \multicolumn{2}{|c|}{ Faixa etária } & \multirow[b]{2}{*}{$40-59$} & \multirow[b]{2}{*}{ Total } \\
\hline & & $15-19$ & $20-39$ & & \\
\hline$\underset{\sim}{\stackrel{\sim}{\sim}}$ & 3 & 19 & 58 & - & 80 \\
\hline$\underset{\sim}{\stackrel{m}{\sim}}$ & 2 & 14 & 66 & 4 & 86 \\
\hline$\stackrel{\sim}{\stackrel{*}{\circ}}$ & 1 & 16 & 57 & - & 74 \\
\hline$\stackrel{\text { Ln }}{\stackrel{\sim}{\sim}}$ & 1 & 20 & 56 & 3 & 80 \\
\hline $\begin{array}{l}0 \\
\stackrel{\circ}{\circ}\end{array}$ & 1 & 12 & 49 & 4 & 66 \\
\hline$\vec{\sim}$ & 1 & 13 & 50 & 2 & 66 \\
\hline $\begin{array}{l}\vec{\pi} \\
\stackrel{0}{0} \\
0\end{array}$ & 9 & 94 & 336 & 13 & 452 \\
\hline
\end{tabular}

Fonte: Elaborada pelos autores.

Realizado em 2011 e 2012, um estudo de amplitude nacional que envolveu 23.894 mulheres nas cinco regiões do país relata resultados próximos aos levantados em Sobral no que diz respeito à faixa etária. Dentre as 16.807 portadoras de sífilis, 70,3\% tinham idade entre 20 e 34 anos $^{16}$.

Outro aspecto que se destaca nos dados epidemiológicos de Sobral é a quantidade de gestantes jovens, com idade entre 10 e 19 anos, infectadas por sífilis. No período de estudo foram registrados 103 casos (22,8\% do total). Um estudo realizado em Minas Gerais reforça a relevância desses números ao afirmar que essa faixa etária de mulheres grávidas apresenta maior chance de pré-natal tardio, o que aumenta o risco de transmissão da doença para o feto, uma vez que essas futuras mães demoram mais tempo para ser diagnosticadas e tratadas ${ }^{17}$.

$\mathrm{Na}$ Figura 2 se observa que a maioria dos casos de SG em Sobral ocorreu entre pessoas pardas (363 casos - $80,3 \%$ do total), seguidas por pessoas brancas (44 casos $-9,7 \%$ do total) e as pessoas negras apresentam um número bem próximo de notificações ( 40 casos $8,8 \%$ do total). Não foi incluído no gráfico, devido ao seu caráter pontual, 1 caso $(0,2 \%$ do total) de SG em pessoa indígena e 2 notificações $(0,4 \%)$ foram ignoradas quanto à cor da pele.

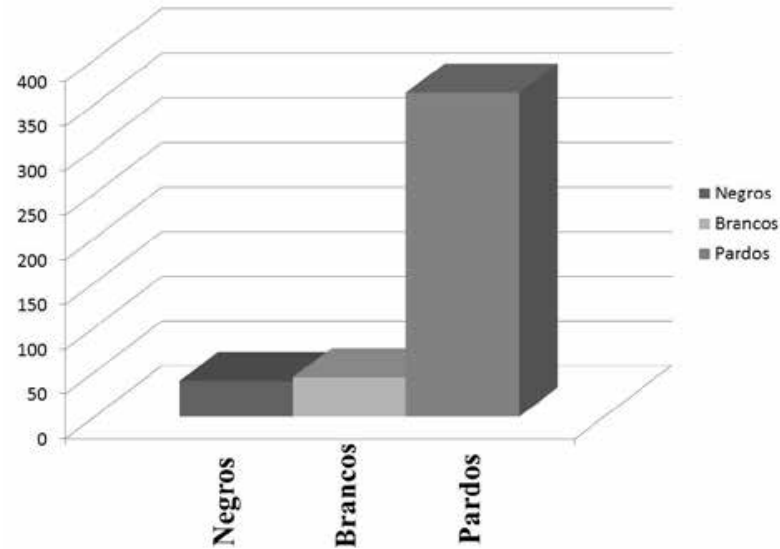

Figura 2. Número de casos de sífilis gestacional por cor da pele. Sobral, 2012-2017.

Fonte: Elaborada pelos autores.

Esses resultados se encontram em consonância com estudos que demonstram as desvantagens enfrentadas por mulheres pardas e negras em relação à assistência à saúde. Elas são vítimas de desigualdade no acesso ao pré-natal adequado, assim como também contam com menor assistência até no momento do parto ${ }^{18}$. Esse problema se relaciona às diferenças socioeconômicas: observam-se piores indicadores de acesso aos serviços de saúde entre pessoas não brancas ${ }^{19}$.

Aspectos que influenciam no acesso ao prénatal e ao parto de qualidade podem influenciar na prevalência de casos de SG entre mulheres pardas em Sobral. Essa hipótese é reforçada quando se evidencia que as mulheres pardas e pretas se concentram mais no Nordeste ${ }^{18}$. Entretanto, há necessidade de novos estudos para embasar cientificamente as iniquidades em saúde no município e suas consequências empíricas.

Em Sobral, a subnotificação de SG entre indígenas decorre de aspectos inerentes a essa clientela, visto que estudos apontam esse contexto em outras regiões do País. No Mato Grosso do Sul, por exemplo, a sífilis apresenta alta incidência entre indígenas, mas a subnotificação nos sistemas oficiais oculta a magnitude da doença ${ }^{20}$. Outra razão para os baixos percentuais observados entre indígenas é o próprio número reduzido dessa população no município ${ }^{12}$.

No que se refere à escolaridade, em Sobral, a SG acomete com maior frequência a população com Ensino Fundamental incompleto (217 casos - $48 \%$ do total). Incluem-se, nesse grupo, os analfabetos e as pessoas que abandonaram os estudos antes de completar a $8^{a}$ série. Diante disso, os dados chamam 
a atenção por corresponderem a quase a metade dos casos notificados no período de estudo, e os números podem ser ainda maiores ao considerar que 44 casos $(9,7 \%)$ foram ignorados em relação ao grau de escolaridade (Tabela 2).

Tabela 2 - Casos de sífilis gestacional por escolaridade. Sobral, 2012-2017

\begin{tabular}{|c|c|c|}
\hline Escolaridade & N & $\%$ \\
\hline Ignorada/em branco & 44 & 9,7 \\
\hline Analfabeto & 14 & 3,1 \\
\hline Ensino Fundamental incompleto (não concluiu da $1^{\text {a }}$ à $4^{\text {a }}$ série) & 42 & 9,3 \\
\hline Ensino Fundamental incompleto (concluiu da $1^{\text {a }}$ à $4^{\text {a }}$ série) & 36 & 8 \\
\hline Ensino Fundamental incompleto (não concluiu da $5^{\text {a }}$ à $8^{\text {a }}$ série) & 125 & 27,6 \\
\hline Ensino Fundamental completo & 61 & 13,6 \\
\hline Ensino Médio incompleto & 62 & 13,8 \\
\hline Ensino Médio completo & 63 & 13,9 \\
\hline Ensino Superior incompleto & 4 & 0,8 \\
\hline Ensino Superior completo & 1 & 0,2 \\
\hline Total & 452 & 100 \\
\hline
\end{tabular}

Fonte: Elaborada pelos autores.

0 maior número de infecções por SG entre mulheres de baixa escolaridade parece relacionarse ao difícil acesso às informações sobre a doença e à maior dificuldade para realização do pré-natal, visto que resultados semelhantes foram relatados em estudo realizado no Rio de Janeiro, o qual avaliou dados das 5 regiões do país e constatou um número 3,2 vezes maior de casos de SG entre mulheres com até 7 anos de estudo em comparação àquelas de maior escolaridade ${ }^{16}$.

Outra análise epidemiológica, realizada em Tocantins, também obteve resultados semelhantes aos de Sobral em termos de escolaridade. Esse estudo indica que $76 \%$ das mulheres com SG variavam entre Ensino Fundamental incompleto e Ensino Médio completo, o que aponta a importância de políticas públicas para orientar essa população de risco para SG sobre a relevância das ações de prevenção e tratamento da doença ${ }^{21}$.

Em relação à zona de residência das gestantes infectadas por sífilis em Sobral, a Figura 3 mostra que houve maior número de casos notificados na zona urbana. 0 número total de notificações na zona rural foi de 73 casos $(16,1 \%)$, ao passo que 377 casos de SG se referem a mulheres que vivem na zona urbana $(83,4 \%)$. Para 2013,2 casos foram ignorados quanto à zona de residência.

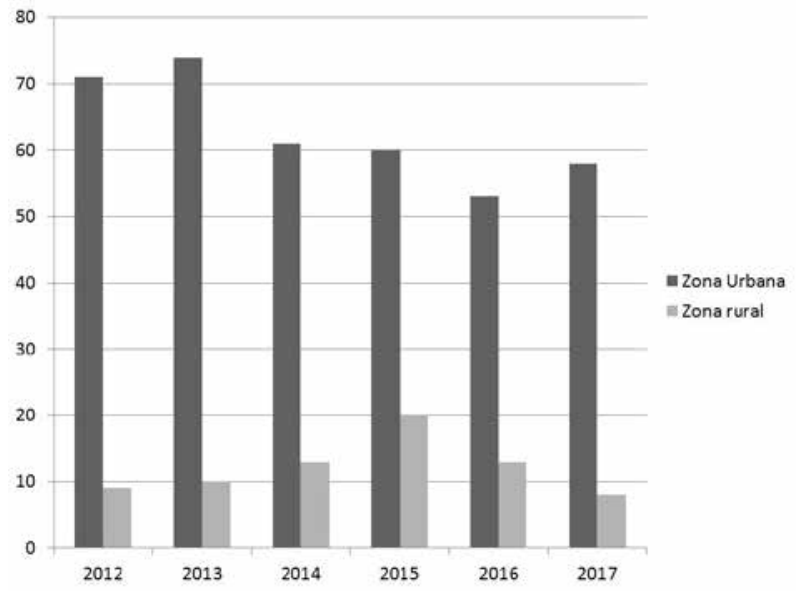

Figura 3. Casos de sífilis gestacional por zona de residência. Sobral, 2012-2017.

Fonte: Elaborada pelos autores.

Esses dados refletem o grande número de pessoas vivendo na zona urbana, característica observada na maioria absoluta das cidades brasileiras. Contudo, um aspecto que assume destaque é a persistência de números relevantes de mulheres com SG na zona rural. Tal fato evidencia que essa população deve ter acesso a políticas de prevenção e tratamento dessa doença, além de iniciativas para promover seu acesso ao pré-natal de qualidade. 
A Tabela 3 mostra predominância da sífilis terciária (341 casos - 75,4\% do total). Outro tipo clínico que demanda atenção é a sífilis latente (66 casos $-14,6 \%)$. A relevância da classificação clínica concerne ao risco de transmissão vertical, que é maior na sífilis primária e secundária ${ }^{1}$.

Tabela 3 - Casos de sifilis gestacional por classificação clínica da doença. Sobral, 2012-2017

\begin{tabular}{lcc}
\hline Classificação clínica & N & $\%$ \\
\hline Ignorada/em branco & 13 & 2,9 \\
Primária & 29 & 6,4 \\
Secundária & 3 & 0,7 \\
Terciária & 341 & 75,4 \\
Latente & 66 & 14,6 \\
\hline Total & 452 & 100 \\
\hline
\end{tabular}

Fonte: Elaborada pelos autores.

Conhecer a caracterização clínica da sífilis é vital para a adoção da terapêtica adequada. Na sífilis terciária e latente tardia, o tratamento demanda uma dose semanal, durante 3 semanas seguidas, enquanto a terapêutica da sífilis primária e secundária envolve uma dose única ${ }^{4}$. Em virtude disso, o tratamento da sífilis terciária e latente tardia apresenta maior taxa de abandono, devido à sua maior complexidade.

Desse modo, como grande parte dos casos notificados em Sobral foi de sífilis terciária, devese focar a terapêtica adequada dos pacientes infectados, visando à redução dos riscos dessa doença.

\section{CONCLUSÃO}

Os resultados deste estudo evidenciam aumento do número de casos de SG em comparação aos dados notificados na década passada. Constatouse predominância de SG com classificação clínica terciária e inclinação para grupos populacionais específicos, como mulheres negras e pardas com idade entre 20 e 39 anos, baixo nível de escolaridade, moradoras da zona urbana e representantes de classes socioeconômicas menos favorecidas.

Diante desse cenário, infere-se que há necessidade de intervenções mais vigorosas voltadas à prevenção, ao diagnóstico precoce e ao tratamento adequado da sífilis, com foco em populações mais vulneráveis, tendo por objetivo diminuir as disparidades sociais observadas.

\section{CONTRIBUIÇÃO DOS AUTORES}

João Vitor Souza Marques contribuiu com a realização da pesquisa, o delineamento do estudo e a redação do manuscrito. Beatriz Mendes Alves contribuiu com a redação e revisão crítica do manuscrito. Marcos Vinícius Souza Marques contribuiu com a realização da pesquisa. Francisco Plácido Nogueira Arcanjo contribuiu com a realização da pesquisa e a revisão crítica do manuscrito. Cynara Carvalho Parente e Renan Lopes Vasconcelos contribuíram com a revisão crítica do manuscrito.

\section{REFERÊNCIAS}

1. Ministério da Saúde. Sífilis [document on the internet]. 2017 [cited 2018 0ct 19]. Available from: http://portalms.saude.gov.br/saude-de-a-z/sifilis-2

2. Secretaria de Estado da Saúde de São Paulo. Sífilis congênita e sífilis na gestação. Rev Saúde Pública [serial on the internet]. 2008 [cited 2018 0ct 20];42(4):768-72. Available from: http://www. scielo.br/pdf/rsp/v42n4/itss.pdf

3. Bruna MHV. Sifilis [document on the internet]. 2018 [cited 2018 0ct 23]. Available from: https:// drauziovarella.uol.com.br/doencas-e-sintomas/ sifilis/

4. Freitas $\mathrm{F}$, Menke $\mathrm{CH}$, Rivoire WA, Passos EP, organizers. Rotinas em ginecologia. São Paulo: Artmed; 2011.

5. Cardoso ARP, Araújo MAL, Cavalcante MS, Frota MA, Melo SP. Análise dos casos de sífilis gestacional e congênita nos anos de 2008 a 2010 em Fortaleza, Ceará, Brasil. Ciênc Saúde Colet [serial on the internet]. 2018. [cited 2018 0ct 22];23(2):56374. Available from: http://www.scielo.br/pdf/csc/ v23n2/1413-8123-csc-23-02-0563.pdf

6. Brasil. Portaria n. 33, de 14 de julho de 2005. Inclui doenças à relação de notificação compulsória, define agravos de notificação imediata e a relação dos resultados laboratoriais que devem ser notificados pelos Laboratórios de Referência Nacional ou Regional. Diário Oficial da União, Brasília (2005 Jul 15); Sec 1.

7. Magalhães Sobrinho DDT. Sífilis gestacional: investigação da fragilidade do tratamento na Estratégia Saúde da Família [document on the internet]. 2017 [cited 2018 Dec 10]. Available from: https://unifor.br/documents/392178/805154/ simposiocienciasmedicas 2017 artigo 34. pdf/54fe38be-4c19-ae78-4fb3-9ca00f55ebf7 
8. Ministério da Saúde. Boletim epidemiológico: sifilis 2017 [document on the internet]. 2017 [cited 2018 Nov 3];48(36):1-44. Available from: http:// portalarquivos.saude.gov.br/images/pdf/2017/ novembro/13/BE-2017-038-Boletim-Sifilis-11-2017publicacao-.pdf

9. TV Diário. Número de casos de sífilis no Ceará cresce $380 \%$ em sete anos [document on the internet]. 2017 [cited 2018 Nov 1]. Available from: http://tvdiario.verdesmares.com.br/noticias/ ciencia-e-saude/numero-de-casos-de-sifilis-noceara-cresce-380-em-sete-anos-saiba-como-seprevenir-1.1840299

10. Soares BGMR, Marinho MAD, Linhares MI, Mota DS. Perfil de notificação de sífilis gestacional e sifilis congênita. Sanare (Sobral, Online) [serial on the internet]. 2017 [cited 2018 Nov 2];16(2):51-9. Available from: https://sanare.emnuvens.com.br/ sanare/article/view/1178/639

11. Sobral (Município). A cidade: história [document on the internet]. 2018 [cited 2018 Nov 2]. Available from: $\quad$ http://www.sobral.ce.gov.br/a-cidade/ historia

12. Instituto Brasileiro de Geografia e Estatística. Cidades: Sobral [document on the internet]. 2018 [cited 2018 Nov 2]. Available from: https://cidades. ibge.gov.br/brasil/ce/sobral/panorama

13. Instituto Brasileiro de Geografia e Estatística. Cidades: Sobral: Serviços de Saúde [document on the internet]. 2019 [cited 2018 Nov 2]. Available from: https://cidades.ibge.gov.br/brasil/ce/sobral/ pesquisa $/ 32 / 28163$

14. Cabral BTV, Dantas JC, Silva JÁ, Oliveira DA. Sífilis em gestante e sífilis congênita: um estudo retrospectivo. Rev Ciênc Plur [serial on the internet]. 2017 [cited 2018 Nov 7];3(3):32-44. Available from: https://periodicos.ufrn.br/rcp/ article/download/13145/9351/

15. Vásquez GGH. Vênus nos braços de mercúrio, bismuto e arsênio: notas históricas sobre sifilis gestacional antes da penicilina. Sex, Salud Soc (Rio J) [serial on the internet]. 2018 [cited 2018 0ct 26];28:226-45. Available from: http://www.scielo. $\mathrm{br} / \mathrm{pdf} / \mathrm{sess} / \mathrm{n} 28 / 1984-6487$-sess-28-226.pdf

16. Domingues RMSM, Szwarcwald CL, Souza Junior PRB, Leal MC. Prevalence of syphilis in pregnancy and prenatal syphilis testing in Brazil: birth in Brazil study. Rev Saúde Pública [serial on the internet]. 2014 [cited 2018 Nov 3];48(5):76674. Available from: http://www.scielo.br/pdf/rsp/ v48n5/pt 0034-8910-rsp-48-5-0766.pdf

17. Jezo RFV, Ribeiro IKS, Araújo A, Rodrigues BA. Gravidez na adolescência: perfil das gestantes e mães adolescentes em uma unidade básica de saúde.
Revista de Enfermagem do Centro-0este Mineiro [serial on the internet]. 2017 [cited 2018 Nov 7];7:1-8. Available from: http://www.seer.ufsj.edu. br/index.php/recom/article/viewFile/1387/1563

18. Leal MC, Gama SGN, Pereira APE, Pacheco VE, Carmo CN, Santos RV. A cor da dor: iniquidades raciais na atenção pré-natal e ao parto no Brasil. Cad Saúde Pública [serial on the internet]. 2017 [cited 2018 Nov 7];33(1):1-17. Available from: http://www.scielo.br/ $\mathrm{pdf} / \mathrm{csp} / \mathrm{v33s} 1 / 1678-4464-\mathrm{csp}-33-\mathrm{s} 1-\mathrm{e} 00078816 . \mathrm{pdf}$

19. Santos JAF. Desigualdade racial de saúde e contexto de classe no Brasil. Revista de Ciências Sociais [serial on the internet]. 2011 [cited 2018 Nov 7];54(1):5-40. Available from: http://www. scielo.br/pdf/dados/v54n1/01.pdf

20. Tiago ZS, Picoli RP, Graeff SVB, Cunha RV, Arantes R. Subnotificação de sífilis em gestantes, congênita e adquirida entre povos indígenas em Mato Grosso do Sul 2011-2014. Epidemiol Serv Saúde [serial on the internet]. 2017 [cited 2018 Nov 7];26(3):50312. Available from: http://www.scielo.br/pdf/ress/ v26n3/2237-9622-ress-26-03-00503.pdf

21. Cavalcante PAM, Pereira RBL, Castro JGD. Sífilis gestacional e congênita em Palmas, Tocantins, 20172014. Epidemiol Serv Saúde [serial on the internet]. 2017 [cited 2018 Nov 9];26(2):255-64. Available from: http://www.scielo.br/pdf/ress/v26n2/22379622-ress-26-02-00255.pdf
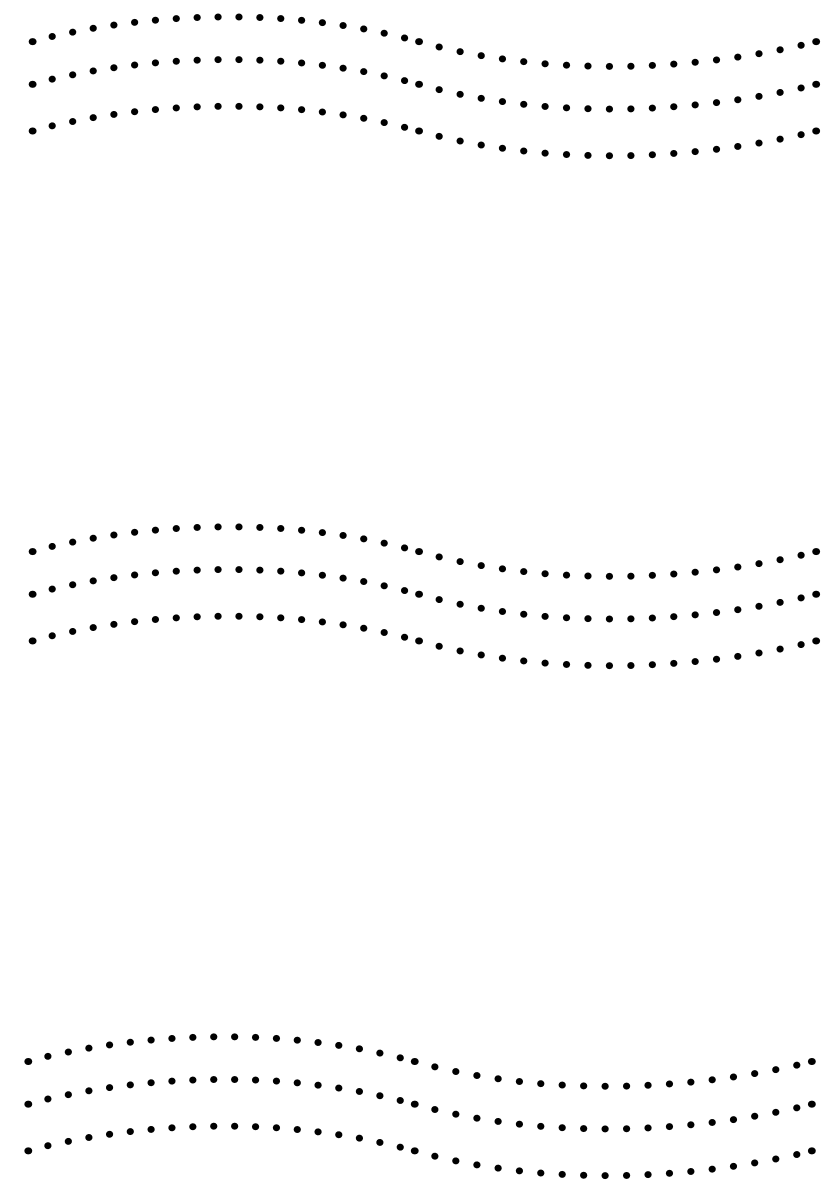